\title{
Experimental Evaluation of the Transmission in a Low Cost SCM/WDM Radio over Fibre System Employing Optical Broadband Sources and Interferometric Structures
}

\author{
Fulvio Grassi, José Mora, Beatriz Ortega, José Capmany \\ ITEAM Research Institute, Universidad Politécnica de Valencia \\ C/ Camino de Vera, s/n 46022 Valencia (Spain) \\ E-mail:jmalmer@iteam.upv.es
}

\begin{abstract}
In this paper, a novel transmission configuration for SCM/WDM radio over fibre communication systems is proposed and experimentally demonstrated. The scheme is based on the spectrum slicing of a low-cost optical broadband source to obtain a set of optical channels compatible with WDM technology. In order to increase the frequency operation range limited to hundreds of $\mathrm{MHz}$ due to the fibre dispersion effects over the optical broadband source, a Mach-Zehnder Interferometric structure is introduced to extend the operative frequency range up to $20 \mathrm{GHz}$. In this context, the quality of service of different digital signals such as BPSK, QPSK, 16-QAM and 32-QAM is experimentally evaluated. The experimental measurements show EVM performances below $6 \%$ for each WDM channel in a standard singlemode fibre link making the architecture suitable for last mile broadband applications in Passive Optical Networks.
\end{abstract}

Keywords: optical communications, multiplexing, passive optical networks, optical broadband sources.

\section{INTRODUCTION}

The current emergence of new ultra-broadband interactive services trough fixed and wireless access networks is favouring the development of Radio over Fibre (RoF) systems due to the high bandwidth offered by the optical media [1]. Furthermore, in order to handle the ever growing data traffic volumes and improve the optical spectral efficiency, the application of optical transmission and networking concepts such as Wavelength Division Multiplexing (WDM) combined with electrical SubCarrier Multiplexing (SCM) schemes, has turned out to be an attractive option for the physical layer infrastructure in Passive Optical Networks (PONs) [2]-[3]. To accelerate a commercial deployment of such architectures a huge progress has been made to reduce their practical complexity. In addition, some efforts have been realized aimed to reduce the high costs of WDM light sources. The introduction of wavelength-locked Fabry-Perot Diodes (FPLDs) with external injection of broadband sources [4], reflective Semiconductor Optical Amplifiers (SOAs) [5] and spectral slicing of incoherent light sources [6] are different approaches proposed to implement a cost-effective solution. We focused our interest on the use of optical broadband sources (OBSs) since spectrum slicing is an attractive WDM technique that provides a low-cost means of sharing a multiwavelength optical source amongst many users. Spectrum slicing of OBS is a strong candidate for fibre-to-the-home access networks and has potential for cost-sensitive local area network applications. However, its limitations in terms of electrical transmission bandwidth and output power still need to be improved especially in the third transmission window where the performance of such links are severely limited by the fibre chromatic dispersion as we have demonstrated theoretically and experimentally in [7].

In this context, we have recently proposed a novel RoF system configuration based on a broadband ASE source and a Mach-Zehnder interferometric (MZI) structure which is able to transport RF signals in an operative frequency range of tens of GHz. We demonstrated that the spectrum slicing of an $80 \mathrm{~nm}$-wide OBS centred at third window into four equivalent optical channels is compatible with a real WDM scenario. The introduction of the MZI permits to obtain a transmission window free from carrier suppression effect (CSE) which can be tuned in a $20 \mathrm{GHz}$ frequency range. In this paper, after a characterization of the electrical transfer function for the experimental approach, we evaluate the overall system capacity through the simultaneous transmission of several digital signals with different modulation formats (BPSK, QPSK, 16-QAM and 32-QAM) in a short-reach standard singlemode fibre (SMF) link. Taking advantage from the tunability of the electrical transfer function, an error-free data transmission with an error vector magnitude (EVM) values below $6 \%$ is achieved in all channels within a $20 \mathrm{GHz}$ RF band. Therefore, the proposed solution opens the perspective to the use of low-cost OBSs as WDM-compatible light sources and shows that bandwidth requirements of a RoF-WDM-PON network for lastmile applications are fulfilled.

\section{EXPERIMENTAL DESCRIPTION OF THE SCM/WDM TRANSMISSION SYSTEM}

Figure 1 illustrates the schematic diagram of the experimental set-up required for downstream transmission of digital signals from a Central Office (CO) to a number of remote Base Stations (BSs). In the CO, an 80 nm-wide OBS centred in the third window is amplitude modulated with SCM data by means of an external electro-optical 
modulator (EOM). Inset (a) of Fig. 1 plots the optical spectrum of the OBS. The modulated optical signal is launched into a SMF link and after propagation a Coarse Wavelength Division Multiplexer (CWDM) slices four optical channels which are almost $17 \mathrm{~nm}$ wide with a wavelength separation of $20 \mathrm{~nm}$ as shown in inset (b) of Fig. 1. The channels are centered at $1531 \mathrm{~nm}, 1551 \mathrm{~nm}, 1571 \mathrm{~nm}$ and $1591 \mathrm{~nm}$ respectively and convey the optical field toward four different BSs. Each BS has a MZI filtering structure before detection which contains a Variable Delay Line (VDL) in one of the two branches of the MZI. In this way, the time delay $\Delta \tau$ between the arms of the MZI can be controlled. As an example, inset (c) of Fig. 1 plots the optical spectra of the four channels at the output of the MZI with a time delay of $12.6 \mathrm{ps}$.

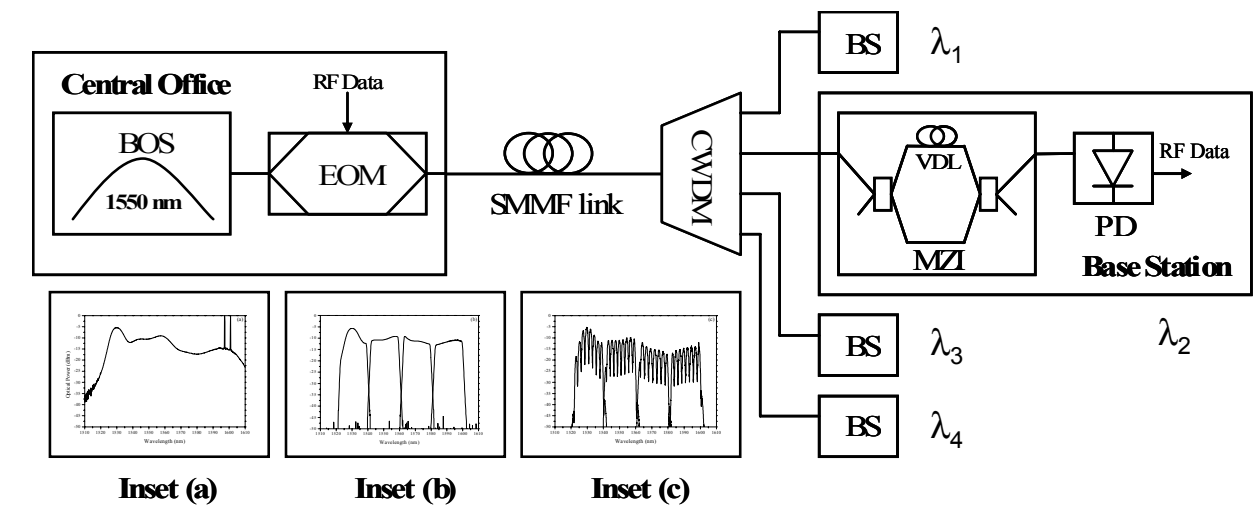

Figure 1. Experimental SCM/WDM fibre radio transport scheme. Insets show the optical spectra of the (a) OBS in CO, (b) each optical channel after CWDM and (c) after the MZI located in each BS

In order to explain the functionality of the MZI located in each BS, Fig. 2 provides the experimental electrical transfer function measured after photodetection for the lower (Fig. 2a) and upper (Fig. 2b) optical channels corresponding to the optical wavelengths given by 1531 and $1591 \mathrm{~nm}$, respectively. In this experiment two different time delays $\Delta \tau_{1}$ (continuous line) and $\Delta \tau_{2}$ (dashed line) are tuned in the MZI. As it can be observed, a baseband (BB) transmission window appears in the electrical transfer function for both optical channels due to the fibre dispersion effects over each $18 \mathrm{~nm}$ optical channel. A lowpass filtering effect is produced giving a BB transmission window with a $3 \mathrm{~dB}$ bandwidth around 0.46 and $0.28 \mathrm{GHz}$ for the lower and upper optical channels, respectively. As we demonstrated previously [7], the use of OBSs in third window reduces drastically the frequency transmission range to few GHz. However, the introduction of the MZI permits to generate new transmission window at higher frequencies. As shown in Fig. 2, apart from BB transmission windows, the electrical transfer function shows two transmission windows which are located at $5 \mathrm{GHz}$ and $15 \mathrm{GHz}$ by selecting two different time delays $\Delta \tau_{1}$ and $\Delta \tau_{2}$, respectively. Therefore, the MZI opens a transmission window by selecting a time delay $\Delta \tau$ whose central frequency is given by the following equation:

$$
f_{0}=\frac{\Delta \tau}{2 \pi \cdot \beta_{2} \cdot L_{\mathrm{FIBER}}}
$$

where $\beta_{2}$ is the chromatic dispersion coefficient and $L_{\mathrm{FIBER}}$ is the length of the optical link. Equation (1) shows that the transmission window can be tuned at different points over the available frequency range with an important increase of the electrical transmission bandwidth. Moreover, this feature adds flexibility to a RoF system and makes it compatible with WDM architectures. In fact in a WDM-PON scenario a point-to-multipoint transmission can be easily enabled once a direct correspondence between the SCM frequency of one service in the $\mathrm{CO}$ and the transmission window of its respective BS is established by tuning the central frequency $f_{0}$.

In addition, we observe that the transmission windows for both optical channels plotted in Fig. 2 have different bandwidths. For a given optical channel, the optical spectrum of the OBS determines the shape of the transmission window. In particular, for the channel centred at $1531 \mathrm{~nm}$ the transmission windows at $5 \mathrm{GHz}$ and $15 \mathrm{GHz}$ have a $3 \mathrm{~dB}$ bandwidth around $0.8 \mathrm{GHz}$, respectively, while the transmission windows corresponding to the optical channel centred at $1591 \mathrm{~nm}$ have a $3 \mathrm{~dB}$ bandwidth of $0.5 \mathrm{GHz}$. However, we have found that the transmission windows obtained for all optical channels are wide enough to extend the operative SCM frequency range from $\mathrm{BB}$ to tens of $\mathrm{GHz}$ as required in $\mathrm{RoF}$ applications. 

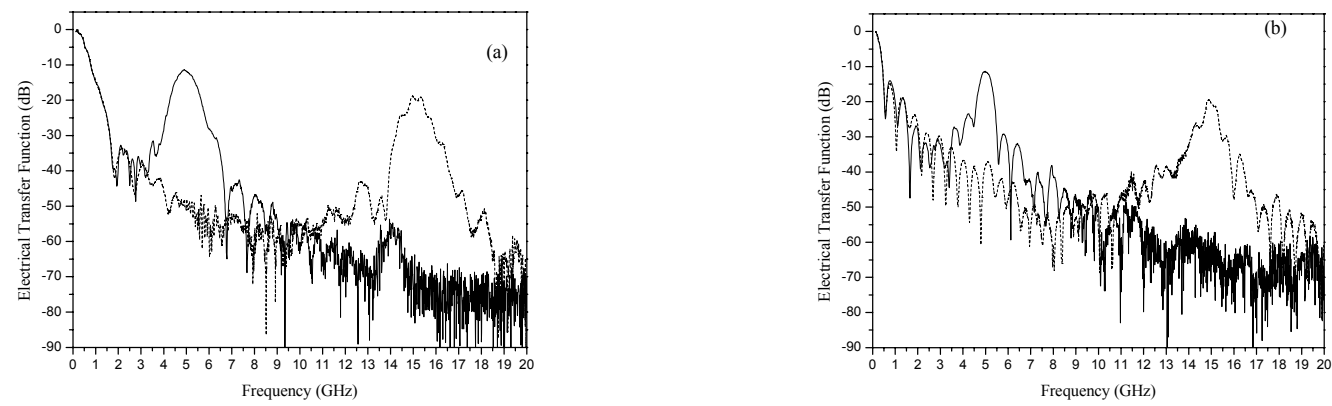

Figure 2. Electrical response at two different optical delays $\Delta \tau_{1}$ (continuous line) and $\Delta \tau_{2}$ (dashed line) when the optical channels (a) $1531 \mathrm{~nm}$ and (b) $1591 \mathrm{~nm}$ are considered.

\section{EXPERIMENTAL EVALUATION OF TRANSMISSION SIGNAL DEGRADATION}

To further demonstrate the capabilities offered by the proposed system we evaluate the transmission degradation of two SCM carriers at $5 \mathrm{GHz}$ and $15 \mathrm{GHz}$ modulated in BPSK, QPSK, 16-QAM and 32-QAM via a $5 \mathrm{Mbps}$ pseudo-random sequence data provided by an Agilent E8267D PSG vector signal generator. At the CO, the RF signals are applied to the EOM with a $3 \mathrm{~dB}-\mathrm{RF}$ bandwidth of $15 \mathrm{GHz}$ in DSB configuration. The optical signal propagates through a $5 \mathrm{~km}$ fibre link and then it is split by a CWDM and delivered to four BS as shown in Fig. 1. By using an Agilent N9020A-526 MXA signal analyser, the electrical subcarriers are finally demodulated.

Evaluating the signal degradation in terms of EVM and using a maximum EVM 10\% criteria [8], a comparison between different modulation formats has been realized. Figures $3 \mathrm{a}$ and $3 \mathrm{~b}$ plots the EVM obtained for each optical channels corresponding with each BS when the electrical subcarriers are $5 \mathrm{GHz}$ and $15 \mathrm{GHz}$, respectively. The experimental results show clearly that an error-free transmission is achieved over the four optical channels. However, for a given RF subcarrier there are some EVM variations when it is measured in different BS due to different optical power distribution between optical channels. The worst case in Fig. 3a is represented by the BPSK modulation format ( $\mathbf{a})$ where the EVM goes from $3.1 \%$ to $4.9 \%$ while the best performance is achieved with 32-QAM $(\boldsymbol{\nabla})$ with EVM from $1.7 \%$ to $3.5 \%$. From Fig. $3 \mathrm{~b}$, we deduce that the transmission is more sensitive to the dispersion slope dependence on the subcarrier frequency according to the peak amplitude difference around $8 \mathrm{~dB}$ in the electrical transfer functions of Fig. 2. In fact a general increasing of the EVM affects all channels although the measured EVM values do not exceed 6\%. Regarding the modulation formats, 16-QAM and 32-QAM achieve higher transmission performances than QPSK and BPSK as expected if we consider that QAM formats utilize the available electrical spectra in a more efficient way than PSK formats.
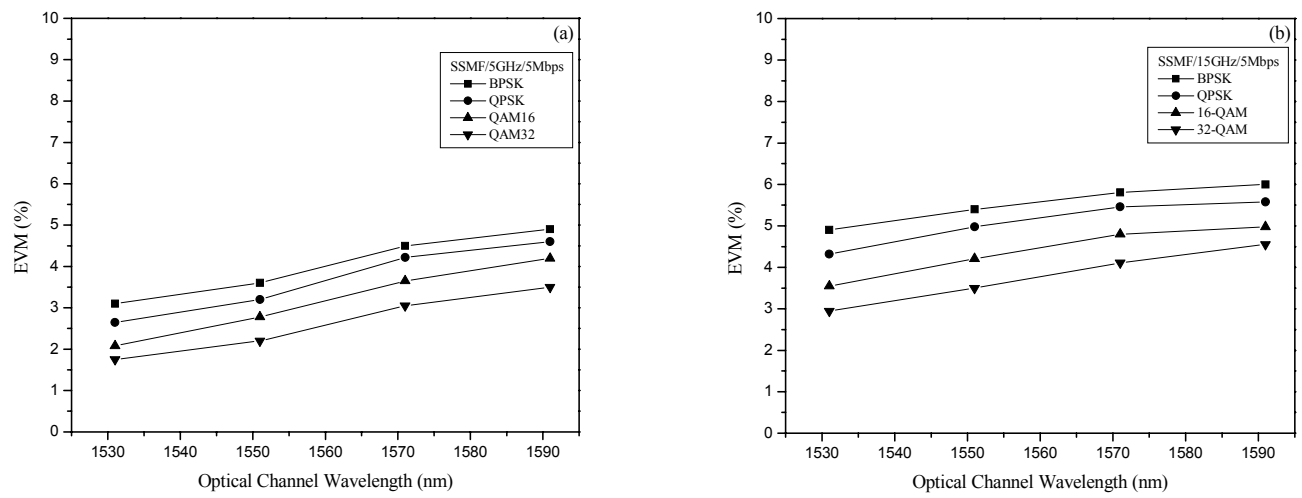

Figure 3. EVM versus channel wavelength for different modulation formats with the electrical subcarrier at (a) $5 \mathrm{GHz}$ and (b) $15 \mathrm{GHz}$.

In Fig. $4 \mathrm{a}$ and $4 \mathrm{~b}$ we illustrate the constellation diagrams captured for subcarrier frequencies at $5 \mathrm{GHz}$ and $15 \mathrm{GHz}$ over the channels centered at $1531 \mathrm{~nm}$ and $1591 \mathrm{~nm}$, respectively. The diagrams show that the transmitted symbols are correctly delivered to the receiver for each channel and subcarrier frequency. 


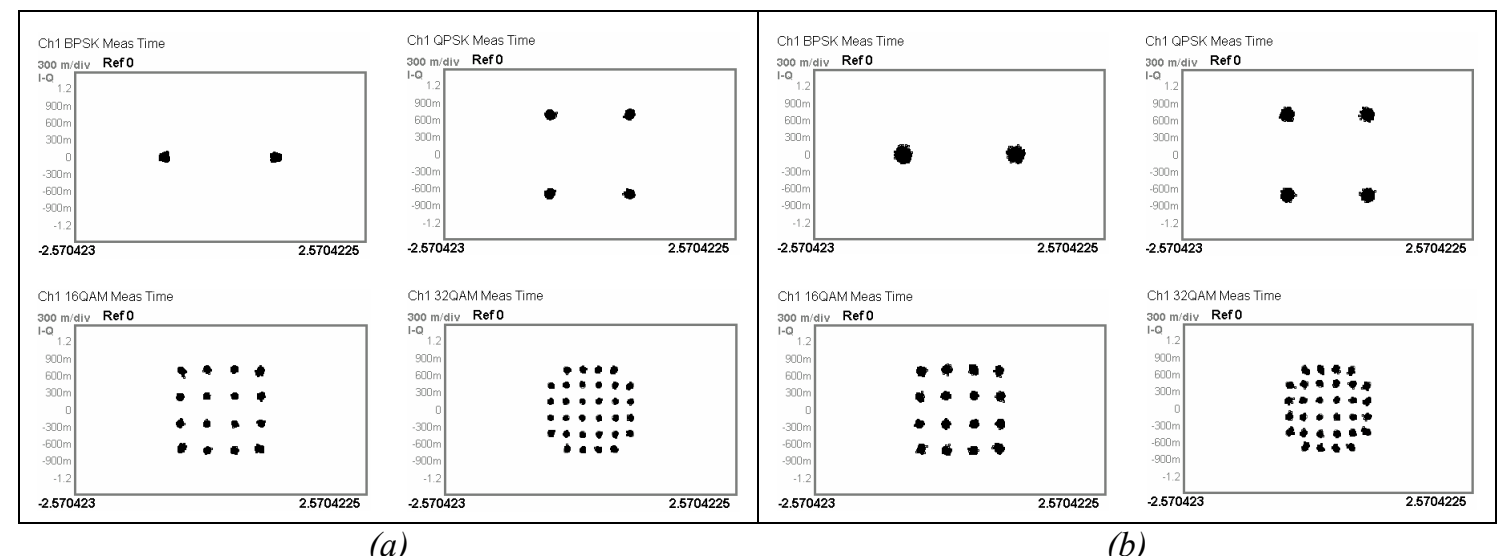

Figure 4. Constellation diagrams for 5 Mbps transmission at different modulation formats (a) channel at $1531 \mathrm{~nm}$ with subcarrier at $5 \mathrm{GHz}$ and (b) optical channel at $1591 \mathrm{~nm}$ with subcarrier at $15 \mathrm{GHz}$.

\section{CONCLUSIONS}

In conclusion, we have presented and experimentally demonstrated a new SCM/WDM optical transmission architecture for broadband applications in current PONs. A spectrum-sliced low-cost OBS centred in third window is used to transport SCM signals up to $20 \mathrm{GHz}$ through four WDM channels. After performing an experimental characterization of the electrical system response for all channels we demonstrate that the introduction of a MZI permits to generate different transmission windows which are also tunable in a wide operating RF range by controlling the optical delay between the arms of the MZI structure. This configuration allows to overcome both BOS bandwidth limitations and fibre dispersion effects which are very critical in the third transmission window region. An end to end demonstration has been also provided by setting up a short-reach experimental transmission over $5 \mathrm{~km}$ of SSMF link. For each channel we have evaluated and compared the system potentialities by transmitting a $5 \mathrm{Mbps}$ pseudo-random data sequence codified in BPSK, QPSK, 16-QAM and 32-QAM formats which modulate electrical subcarriers at $5 \mathrm{GHz}$, and $15 \mathrm{GHz}$. The EVM values obtained are largely below the maximum $10 \%$ with the best performances achieved with QAM modulation formats followed by QPSK and BPSK. Although a different power distribution between channels causes a small EVM increase with the optical wavelength, the OBS spectral slicing combined with the MZI structure improves successfully the RF operative bandwidth of the system. These two key results demonstrate that the proposed system is compatible with WDM technology and fulfils the requirements of a RoF link in order to accelerate the employment of OBS in actual low-cost WDM-PONs.

\section{ACKNOWLEDGEMENTS}

The authors wish to acknowledge the European Commission FP7 under project ALPHA (grant no. 212352) and PROMETEO GVA 2008-092 Microwave Photonics Research Excellence Project supported by the Generalitat Valenciana.

\section{REFERENCES}

[1] L.G. Kazovsky et al.: Next-generation optical access networks, J. Lightwave Technol., vol. 25, pp. 3428-3442, Nov. 2007.

[2] C.-H Lee, et al.: Fibre to the home using a PON infrastructure, J. Lightwave Technology, vol. 24, pp. 4568- 4583, Dec. 2006.

[3] R. Lin: Next generation PON in emerging networks, in Proc. OFC 2008, San Diego (CA), Feb. 2008, paper OWH1.

[4] P.K.J. Park et al.: Reduction of polarization-induced performance degradation in WDM PON utilizing MQW-SLD-based broadband source, Opt. Express, vol. 21, pp. 14228-14233, Oct. 2007.

[5] K. Y. Cho et al.: 10-Gb/s operation of RSOA for WDM PON, IEEE Photon. Technol. Lett. vol. 20, pp. 1533-1535, Sept. 2008.

[6] J. Cho et al.: Broadcast transmission in WDM-PON using a broadband light source, in Proc. OFC 2007, Anaheim (CA), Mar. 2007, paper OWS7.

[7] F. Grassi et al.: Subcarrier multiplexing tolerant dispersion transmission system employing optical broadband sources, Opt. Express, vol. 17, pp. 4740-4751, Mar. 2009.

[8] C. Lethien et al:: Potentials of radio over multimode fibre systems for the in-buildings coverage of mobile and wireless LAN applications, IEEE Photon. Technol. Lett., vol. 17, pp. 2793-2795, Dec. 2005. 